\title{
The Role of Person-Environment Fit in Employee's Working Quality and Job Burnout: A Case of the West-Mazandaran Power Distribution
}

\author{
Parisa Riahifar \\ Department of executive management, Ayatollah Amoli Branch, Islamic Azad University, Amol, Iran \\ Professor Iraj Mahdavi
}

Department of industrial engineering, Mazandaran university of Science and Technology, Babol, Iran

Doi:10.5901/mjss.2016.v7n3s2p223

\begin{abstract}
Job or career compatibility is one the key factors for one's success in the workplace. When there is a good fit between individual and his/her work environment, higher levels of satisfaction and mental and physical well-being are achieved. Personorganization fit has important job-related consequences, including organizational assimilation, job selection, job satisfaction, organizational commitment and desertion. The purpose of this research is to examine the relationship of person-environment fit with employee's working and job burnout in West-Mazandaran Power Distribution Company. This is an applied research conducted based on a correlational design. The actual data was collected from the questionnaires distributed among the company employees. The target statistical population included all employees of the above mentioned company (150 in total) from among whom, using the Krejcie and Morgan table for sample size, 108 employees were selected as the sample. The hypothesized relationships between the variables were investigated based on the information obtained from the standard questionnaires whose validity and reliability had been verified in advance. Data analysis included both descriptive and inferential statistics performed in the SPSS environment. The results indicated a positive and significant relationship between person-environment fit and the employee job burnout. In addition, data analysis showed that person-environment fit did contribute to the employee's working quality in the understudy power company.
\end{abstract}

Keywords: person-environment fit, working quality, job burnout, job compatibility

\section{Introduction}

Human resource strategic management is focused on the internal consistency and coordination of the human resource management actions for building of the employee knowledge, skills and capabilities which are meant to support the competitive strategies and accomplishment of the business objectives. Person-environment fit plays a significant role in human resource management (HRM), as it becomes the main link between horizontal and vertical alignment within strategic HRM, and creates a base enabling achievement of competitive advantages (Marchington and Wilkinson, 2008). Person-environment fit can be used for directing the course of HRM development and creating consistent messages which are shared by managers in the organization (Hall and Hall, 1985).

Studies of person-environment fit suggest three types of fit. The first, person-job fit, is the oldest and the most discussed one in the HRM texts. The concept person-job fit involves compatibility of the employee skills, knowledge, and abilities with performance of particular tasks at work (Edwards, 1994). Person-environment fit also includes person-group and person-organization fit. Person-group fit identifies the supplementary aspects of compatibility for successful functioning with colleagues in a work group or team (Werbel and Gilliland, 1999). Person-organization fit concerns compatibility of the employee interests, values and needs with organizational culture (Chatman, 1989).

Years after, Jansen and Kristof-Brown (2006) proposed a multidimensional typology of person-environment fit which outlined various types of fit between a particular personal variable and a work-related feature. In their typology, though, they proposed direct evaluation of the fit without interpreting the structures which are constituents of the fit (Kristof-Brown, Johnson, and Colbert, 2002).

When there is no fit between personal and organizational characteristics, that is absence of compatibility between individual competences and job requirements, people and organizations would not operate effectively and efficiently. Organizations seek to recruit the ones who better meet the job requirements, are better adapted with training and changes in job require, and remain committed and loyal to organization. 
In today's societies, competitiveness has become vital to all service organizations on economic enterprises, and for their survival, they need to keep with their congeners in competitiveness. Crucial for organization survival is retention of the current customers and attracting the new ones. Since attraction of new customers is far more difficult than retention of the existing ones, creating loyalty in customers by providing them the expected and right services which correspond to their potential needs is conducive and likely to produce the desired effect. Because failing to secure the customer satisfaction with quality of the provided services, we should expect their imminent switching to the rival enterprises and deterioration of our expected resources. This gives rise to the issue of the employee's working quality in organization.

Job burnout is a state of the cumulative tension or stress caused by job-related factors throwing person off his/her mental and physical balance, which is accompanied with three major signs of personal inefficiency, depersonalization, and emotional exhaustion (Fouladband, 2006). Job burnout is a result of prolonged stress and it is estimated that 50 to 80 percent of all diseases are caused by stressful factors and situations (Becker and Huselid, 2006). Job burnout is not simply the fatigue and the stress caused by continuous working, but rather affects person's lifestyle and his hours off duty. Also the pressures produced from passive relationship with friends and acquaintance may lead to one's exhaustion. Thus, the mental health in all areas of person's life, including his working life, is very important. Some studies report a positive relationship between elements of job burnout and the tendency to leave the job. The result of job burnout is loss of motivation, lack enthusiasm and energy, and reduced functioning in life (Sarvari, 2009).

Now, the question is as to what extent the person-environment fit affects the employee's quality of work and job burnout. To answer this question, given the importance of the topic and the above argument, this study attempts to find out how significant is the role person-environment fit in working quality and job burnout of the employees in the mentioned organization.

\section{Literature Review}

Person-environment fit: person-environment fit, in a work setting, involves creating a fit between employee's values, skills, knowledge, and conduct and his work environment. This fit is of certain advantages both to employers and employees (Hitt et al, 1998).

Person-job fit: refers to compatibility of the employee's skills, knowledge, capabilities with performance of certain job tasks in a work context (Edwards, 1994: 249).

Person-group fit: identifies the complementary aspects of compatibility for successful working with colleagues in a work group or team (Werbel and Gilliland, 1999: 249).

Person-organization fit: concerns the compatibility of the employee's interests, values, and needs with organizational culture (Chatman, 1989: 249).

Working quality: all the activities, processes, and courses of action initiated to solve the customer problems. The official definition of quality as given by the American Society for Quality (ASQ) is "the totality of features and characteristics of a product or service that bears on its ability to satisfy stated or implied needs." And the attributes recognized for service quality are: timeliness, completeness, satisfying, consistency (reliability), availability, safety, and responsiveness (Sayed Javadin, 2002).

Job burnout: is a state of cumulative tension or stress caused by certain job-related factors throwing person off his mental and physical balance. It is characterized by three major symptoms of personal inefficiency, depersonalization, emotional exhaustion (Fouladband, 2006).

Girman et al (2013) examined the relationship of human capital with production quality and economic growth in 31 African nations for the period 1975 through to 2008. Their results indicated that the growth in physical capital accounted for 67 percent of GDP, whereas the GDP share of the human capital in these countries was only 22 percent. As for the production quality, 46 percent improvement thereof came from an increase in the human capital.

Olayemi (2012) examined the relationship between human capital investment and industrial productivity in Nigeria, using secondary datasets from the period 1978-2008. His findings indicated that the government's spending on human capital and its training has enduring association with industrial productivity. Chen and Kao (2012) using questionnaire conducted a survey on the effects of job burnout on performance of the firefighters. They found that job burnout could mediate between job demands and health problems. They also reported that the problems associated to job burnout could negatively affect performance of the firefighters. Abdullah (2011), using Maslach Burnout Inventory (MBI), investigated the impact of job burnout on job performance among the nurses of a private hospital in Perak city, Malaysia. He documented a significant relationship between job burnout and performance of the employees. In addition, he demonstrated that emotional exhaustion and depersonalization significantly contributed to individual performance among the understudy nurses. Hong and Shih (2010) examined the impact of industrial knowledge management and 
environmental strategy on corporate performance of the ISO14000 companies in Taiwan. In this study, the data was collected through the questionnaire and, applying structural equation modeling (SEM) technique, was subsequently analyzed for test of the research hypotheses. The results indicated that environmental strategy was positively associated with knowledge flow process and group performance, and knowledge flow process, in turn, was found to be positively related with performance of the whole organization.

\section{Methodology}

This study is an applied research which was conducted according to a descriptive-correlational survey design. Correlational in that it seeks to find out about the relationship between the involved variables. The target statistical population included all employees of the West-Mazandaran Power Distribution Company (150 in total). From among this population, based on the Krejcie and Morgan table for sample size and using simple random sampling, 108 employees were selected as the research sample. To obtain the required actual information, it was made use of three standard questionnaires graded on a 5-point Likert scale.

\section{The Conceptual Model}

Based on the relevant theoretical literature, the research conceptual model was designed, as is represented in figure 1 below.

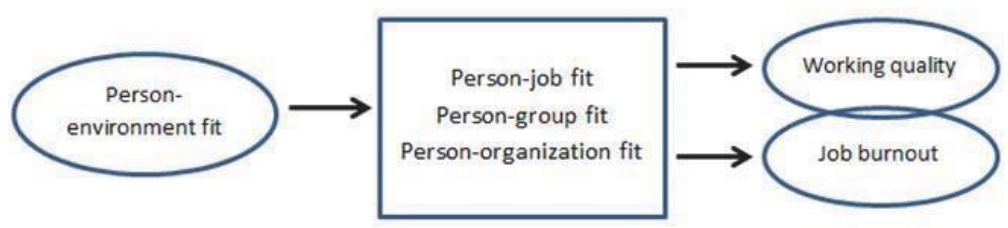

Figure 1 - The conceptual model on the relationships between the involved variables

Source: Musiol \& Boehnke, 2013; Peltokangas, 2014; Malik \& Misra, 2014; Werber \& DeMarie, 2005

\section{Research Hypotheses}

(a) The main hypotheses:

1. Person-environment fit has impact on the employee working quality in West-Mazandaran Power Distribution Company.

2. Person-environment fit has impact on the employee job burnout in West-Mazandaran Power Distribution Company.

(b) The sub-hypotheses:

1. Person-job fit has effect on the employee working quality in West-Mazandaran Power Distribution Company.

2. Person-group fit has effect on the employee working quality in West-Mazandaran Power Distribution Company.

3. Person-organization fit has effect on the employee working quality in West-Mazandaran Power Distribution Company.

4. Person-job fit has effect on the employee job burnout in West-Mazandaran Power Distribution Company.

5. Person-group fit has effect on the employee job burnout in West-Mazandaran Power Distribution Company.

6. Person-organization fit has effect on the employee job burnout in West-Mazandaran Power Distribution Company.

\section{The Results}

Data analysis was performed in the SPSS software. Before test of the hypotheses, the Kolmogorov-Smirnov (K-S) test of 
normality is performed to see if the data regarding the variables are normally distributed or not, based on which the appropriate procedure is chosen for test of the hypotheses. Table 1 summarizes the results of the K-S test.

Table 1 - The results on K-S test of normality $(n=108)$

\begin{tabular}{lcc}
\hline Variable & Test statistic & Sig \\
\hline Job burnout & 0.614 & 0.864 \\
Working quality & 0.711 & 0.693 \\
Person-organization fit & 2.47 & 0.001 \\
Person-job fit & 2.31 & 0.001 \\
Person-group fit & 2.23 & 0.001 \\
Person-environment fit & 2.05 & 0.001 \\
\hline
\end{tabular}

As is seen in table 1, the test statistic for most of the variables does not lie between the range of -1.96 and +1.96 , thus the null hypothesis on normal distribution of the observations is rejected. Therefore, Spearman correlation is used for test of the hypotheses the results of which are summarized in table 2.

Table 2 - The results of Spearman correlation test ( 0.05 level of error)

\begin{tabular}{lccl}
\hline Variable & Correlation coefficient & Sig & Result \\
\hline Person-environ fit \& working quality & 0.380 & 0.037 & $H_{0}$ rejection \\
Person-environ fit \& job burnout & -0.377 & 0.009 & $\mathrm{H}_{0}$ rejection \\
Person-job fit \& working quality & 0.353 & 0.018 & $\mathrm{H}_{0}$ rejection \\
Person-group fit \& working quality & 0.319 & 0.025 & $\mathrm{H}_{0}$ rejection \\
Person-organization fit \& W. quality & 0.321 & 0.028 & $\mathrm{H}_{0}$ rejection \\
Person-job fit \& job burnout & -0.372 & 0.007 & $\mathrm{H}_{0}$ rejection \\
Person-group fit \& job burnout & -0.292 & 0.009 & $\mathrm{H}_{0}$ rejection \\
Person-organization fit \& job burnout & -0.378 & 0.01 & $\mathrm{H}_{0}$ rejection \\
\hline
\end{tabular}

As is seen from the SPSS output in table 2, the obtained level of significance for each and every dimension of personenvironment fit is smaller than 0.05 , so the null hypothesis $\left(\mathrm{H}_{0}\right)$ is not confirmed. It is, accordingly, inferred that, at 95 percent confidence, person-environment fit and its triple dimensions - i.e. person-job fit, person-group fit, and personorganization fit - are positively associated with the employee's working quality, while with the employee's job burnout they stand in negative relation.

\section{Conclusions}

The results showed that person-environment fit did have effect on working quality of the employees in West-Mazandaran Power Distribution Company. Since the correlation coefficient hereof was positive, the relationship was a direct one. The same results were obtained by Hajilu (2011) who investigated the relationship of job stress, job burnout, and quality of working life of the employees with their satisfaction with the organizational climate at the University of Mohaghegh Ardabili and reported that person-environment fit can enhance employee's working quality.

In addition, the results indicated that person-environment fit is inversely associated with the employee's job burnout in the understudy company. This result is similar to that found by Rantakokko et al (2013).

With regard to the constituents of person-environment fit, person-job fit was found to be of influence in the employee's working quality in the mentioned company. And given positiveness of the correlation coefficient, the relationship hereof is of a direct one. This result is consistent with that found by Werbel and DeMarie (2005) in their study, Aligning Strategic HRM and Person-Environment Fit, provide evidence indicating that person-job fit affected the employee's working quality.

As for the person-group fit, the results confirmed its effect on the employee's working quality in the mentioned power company. And given the positive correlation coefficient hereof, the relationship of a direct one. This result is consistent with the findings of Farahmand (2006).

Person-organization fit was also found to be of influence in the employee's working quality in this organization. Likewise, the correlation coefficient hereof was positive which implied a direct relationship. This finding was in line with the result obtained by Huang and Shih (2010). 
Further, the results suggested that person-job fit was inversely related to the employee's burnout in the mentioned organization. Consistent with this result, Shojaee et al (2008) reported that person-job fit negatively affected the employee's burnout.

Moreover, the results indicated the inverse effect of person-group fit on the employee's burnout in the understudy power company. This finding is consistent with the result documented by Grayson and Alvarez (2007) who investigated the relationship of the school organizational climate and the job burnout of the Mexican teachers.

Finally, according to the results, person-organization fit and the employee's burnout were inversely correlated with each other. Similar result was found by Davis and Lofquist (1984).

\section{Suggestions}

Considering the obtained results from this study, the following suggestions are made:

- The significant impact of person-environment fit and its components on the employee's working quality in West-Mazandaran Power Distribution Company is likely to be the case in nearly all organizations and companies. It implies that this category of job-related fits should be taken serious in all enterprises and corporations and this fit and compatibility managers can play a very important role.

- The inverse impact of person-environment fit and its components on the employee's job burnout observed in this study implies necessity of certain changes in organizations for creating an organizational condition which enables the employees to better adapt themselves with organizational environment and other employees reducing thereby the employee job burnout.

\section{References}

Sarvari, Mohammad-Hossein (2009), The Fatigue and Work Psychological Condition; Case Study: Craftsmen of Isfahan Province, psychological Studies, Faculty of Education Sciences and Psychology, Alzahra University, 4(1): 22-32

Seyyed Javadin, Reza (2002), The Principles and Applications of Human Resource Management and the Employee Affairs, Negahe Danesh Publications

Fouladband, Farzaneh (2006), Job Fatigue: The Causes and Solutions, The Health Weekly, 3(106): 8

Olayemi, S.O. (2012), Human Capital Investment and Industrial Productivity in Nigeria, International Journal of Humanities and Social Science, 2(16): 298-307

Werber, J. \& DeMarie, S. (2005), Aligning Strategic HRM and Person - Environment Fit, Human Resource Review, 15,247-267 